\title{
Desarrollo neuropsíquico del lactante
}

\author{
Extracto y comentario al artículo publicado en Rev Chil Pediatr 1934; 5: 648-57
}

\author{
Autor: Manuel Zorrilla \\ Comentario al artículo: LUISA SCHONHAUT B. ${ }^{1}$ \\ 1. Departamento de Pediatría, Facultad de Medicina Clínica Alemana - Universidad del Desarrollo.
}

Palabras claves: Desarrollo, pediatría, historia pediatría.

Key words: Development, pediatrics, pediatric history.

\section{Introducción}

En 1928, bajo el gobierno del General Carlos Ibáñez del Campo, fue promulgada la Ley de Protección de Menores, en que se promovió el derecho al cuidado físico, a la educación y a la consideración social, y, junto con ello, se crearon una serie de instituciones de responsabilidad estatal para la acogida para niños en situación irregular ${ }^{1,2}$.

En aquellos años, además de los elevadísimos niveles de mortalidad infantil y desnutrición, eran frecuentes los retrasos del desarrollo y déficits cognitivos, especialmente en los niños abandonados, pero también en los institucionalizados. Si bien la prioridad era salvar a los pequeños, en la medida que sobrevivían y alcanzaban la adultez, fue aumentando la alerta en relación a la pesquisa e intervención de los déficits $^{3,4}$. Esta preocupación quedaría registrada por los doctores Luis Calvo Mackenna y Manuel Zorrilla en los años 30-40 “...si bien la institución puede ya entregar a la sociedad niños en buen estado físico, no puede decirse lo mismo en lo que respecta a su capacidad de adaptación y a su utilidad social"s. "Cuando un huérfano asilado llega a la adolescencia o a la edad adulta, y cuando abandona el orfanato y se incorpora en la sociedad en que debe ganar su vida, sufre el más trágico de los trastornos sentimentales y morales. La realidad muerde con dientes de fuego"3.

Hace un poco más de 75 años, siguiendo una metodología de observación directa, Zorrilla, con gran fineza semiológica, describía los hitos que iban adquiriendo los 30 niños que estaban a su cargo en la Casa Nacional "con el objeto de poder apreciar en qué medida esta alterado el desarrollo neuropsíquico del niño recluido, niño que físicamente presenta una inferioridad de todos conocida, no siempre explicable" y pese a que consideraba que "en la mayoría de los casos muy difícil de modificar", era destacable los logros que iban alcanzando los pequeños a medida que se mejoraban las condiciones de su entorno.

\section{Artículo de Manuel Zorrilla M.}

Este trabajo fue iniciado con el objeto de poder apreciar en que medida está alterado el

Recibido el 20 de enero de 2014, aceptado para publicación el 03 de frebrero de 2014.

Correspondencia a:

Luisa Schonhaut Berman

E-mail: Ischonhaut@alemana.cl 
desarrollo neuropsíquico del niño recluido, niño que físicamente presenta una inferioridad de todos conocida, no siempre explicable y en la mayoría de los casos muy difícil de modificar.

Sobre desarrollo neuropsíquico del lactante se han preocupado investigadores, pedagogos y médicos, pero, las tablas de examen basadas en sus observaciones, no siempre concuerdan y contienen a menudo pruebas de difícil manejo. El trabajo que nos dimos primero, fue entonces, tratar de aislar las pruebas más sencillas y que mejor hicieran destacar el desarrollo de las diversas funciones.

Daré a continuación un resumen de esas apreciaciones sobre el desarrollo, las que me han parecido más sobresalientes.

La primera manifestación del niño al nacer es el grito. No se puede hablar de verdadero llanto por no haber salida de lágrimas. Estos gritos serian reflejos congénitos producidos por la sensación desagradable de frío y por la sensación de asfixia al interrumpirse la circulación fetal. Cuando el niño se abriga, deja de llorar.

Los primeros días duerme sin interrupción, y se despierta con gritos para alimentarse. En los mementos de vigilia efectúa movimientos inconexos, arrítmicos, como los de un anencéfalo. No son precisamente reflejos y lo curioso es que son ellos los que modificándose, dan lugar a los movimientos expresivos del lactante mayor.

Otros movimientos que efectúa perfectamente desde el nacimiento son los reflejos elementales, que también existen en los anencéfalos, Citaremos los movimientos de deglución, de chupar, de bostezar, estornudo, vómitos, convulsiones, movimientos de prehensión, etc. Hay que agregar a este grupo también los reflejos internos de la vida vegetativa y ciertos reflejos provocables como el palpebral al tocarle la nariz o soplarlo, el Babinski reflejo plantar, los reflejos de defensa, los de succión, al rozarle zonas vecinas a los labios, etc. Otros movimientos que observamos en el recién nacido son los llamados congénitos instintivos, heredados, en los cuales parece que apuntara la conciencia; pero que en realidad son independientes de ella. Acompañan la satisfacción de actos vitales. Por ej., los movimientos de la cabeza y de la boca para buscar el pecho, o bien el niño saciado que se retira del pecho. Si a un lactante pequeño se le cambia su alimento acostumbrado, no chupa; es decir, aparecen estos movimientos como rodeando un reflejo elemental, cual es el de la succión.

Más o menos al mes de edad, aparecen las llamadas reacciones adquiridas elementales, vecinas a los reflejos; pero un poco más complicadas, ya que exigen un substrato anatómico más perfecto. Ejs.: oclusión palpebral al aproximarle un objeto a los parpados; fijación de la mirada; el acto de restregarse cuando se le roza; intensificar los movimientos de succión cuando hay alguna dificultad a la salida del alimento etc.

Se ha llamado síndrome neuropsíquico del recién nacido a aquel caracterizado por: viveza de los reflejos tendinosos, el Babinski, la tendencia a mantenerse en actitudes provocadas o catatonia y los movimientos automáticos que aparecen en una parte del cuerpo, cuando se le provoca en otras o sincinesias y la alteración del tonus muscular.

Respecto a las sensaciones, no las tiene en verdadero sentido, puesto que sin la intervención del cerebro, no puede haber proceso subjetivo. No hay pues, percepciones.

Al nacer no parpadea; pero ya al segundo día si al niño se le acerca una luz intensa cierra los ojos. Los movimientos de los globos oculares no son coordinados. Existe en el recién nacido una ceguera psíquica. Convergencia se observa de la $2^{\circ}$ a la $3^{\circ}$ semana, siempre que se intercepte la mirada en el eje visual. Durante el primer mes, el campo visual es tubular. Las primeras dos semanas tiene fotofobia. A la $5^{\circ}$ semana acomoda la visión a un objeto brillante. A la $8^{\circ}$ semana busca dentro del campo visual un objeto luminoso.

Respecto al oído: sólo después del $2^{\circ}$ ó $4^{\circ}$ día se hace sensible a los ruidos intensos y bruscos, fecha en que la caja del tímpano se ha limpiado y contiene aire. El recién nacido sufre también de sordera psíquica y los primeros días el sonido le provoca reflejo palpebral. Los primeros signos de audición se manifiestan por la mímica: cierta inmovilidad en los rasgos, con atención vaga a la voz. Ocurre esto entre la 
$2^{\circ}$ y $3^{\circ}$ semana de la vida. A los 2 meses busca el ruido y lo localiza.

El olfato parece ser uno de los sentidos que más tarde se despiertan (más o menos al año). En cambio, el gusto aparece ya al nacer, y aún en los prematuros. La mímica del gusto también es congénita. Lo dulce le provoca movimientos de succión; el vinagre, la sal, movimientos de repulsión. Tampoco se puede hablar de una verdadera percepción gustativa.

Las vías sensitivas están muy poco desarrolladas en el recién nacido, corno lo prueba el escaso dolor que le produce la excitabilidad eléctrica. En el primer semestre faltan las localizaciones de sensaciones desagradables, de calor o frío. El máximo de sensación está en la punta de la lengua, donde determina movimientos de succión; más atrás, produce reflejo nauseoso.

Las sensaciones generales (de cansancio, hambre. sed), están desarrolladas congénitamente y varían muy poco en su expresión.

A la $3^{\circ}$ ó $4^{\circ}$ semana hay signos de memoria. Sabe cuándo le toca la comida y quién se la da. Un niño de 3 semanas parece olvidar, al cabo de 4 días de alimentarse con biberón, el pecho que tanto costó que abandonara. A los 4 meses desconoce a los padres que dejo de ver sólo 5 días. En el segundo semestre basta una semana para que se haya olvidado un recuerdo. Al año y medio rememora cosas ocurridas 2 y 3 semanas atrás. A partir de esta edad, la memoria se perfecciona rápidamente con la repetición de las sensaciones. Lo afectivo es lo que recuerda por más tiempo (visitas desagradables del médico). La memoria mecánica es más tardía (3 años), edad en que puede aprender una poesía sin comprenderla.

El niño nace sin atención; a la $4^{\circ}$ semana, más o menos, hay atención involuntaria (mira, toca, etc.) que dura poco tiempo, pero que en compensación la recupera después de un corto descanso. Con la aparición de la atención y la memoria puede decirse que nace la inteligencia.

Hacia el $4^{\circ}$ mes se efectúa una función activa de los sentidos, como es el juego. A los 6 meses mueve las manos bajo el control de la vista. A los 7 meses puede sentarse y con esto ha conquistado el espacio inmediato. El espacio lejano lo tendrá cuando gatee y ande.
Sin embargo, antes del año y medio no hay verdadero concepto de espacio. El primer concepto especial que adquiere es el de su cuerpo; hasta entonces le da de comer a su pie y puede lastimarse o morderse.

La afectividad del lactante es eminentemente egoísta. Gira alrededor de su propio bienestar o dolor. Los estados afectuosos en el son pasajeros e inconstantes (cólera, espanto, alegría, etc.). Sólo en la niñez comenzaran a aparecer las emociones prolongadas y menos intensas como el abatimiento, la opresión, etc.

Respecto al lenguaje infantil, se acepta que existe una actividad congénita ancestral instintiva (instinto del lenguaje) en la que prende fácilmente la imitación. De ahí que un sordo mudo sea mudo porque es sordo a pesar de que puede gritar y modular ciertos sonidos.

La imitación se manifiesta alrededor de los 2 años. La espontaneidad, entre 2 y 3 años. El lactante pequeño expresa sus sensaciones con gritos más o menos parecidos. A los 2 meses consigue el balbuceo, que adquiere el carácter de un verdadero juego. Gritos y balbuceos son independientes del desarrollo auditivo. Se los observa también en los sordos mudos.

Entre el $6^{\circ}$ y $7^{\circ}$ mes, comienza la imitación de lo escuchado. Primero se imita a sí mismo. Entre el $8^{\circ}$ y $9^{\circ}$, la imitación de palabras sencillas cuyo significado no comprende (papá, mamá). Es este el período de papagallo.

En sus primeras palabras, el niño sigue la ley del menor esfuerzo. Usa sobre todo la articulación de los labios y la punta de la lengua; duplica las silabas, etc. Si a veces se le escucha articular palabras más difíciles, se debe a la enseñanza de sus familiares. Las palabras raras son a menudo mutilaciones de palabras oídas. Después del año, viene el periodo de comprensión. Primero el de los gestos y ademanes, llora cuando se le amenaza, si se le pregunta dónde está su madre, la mira o señala. Por entonces se ayuda el niño en su expresión con el lenguaje mímico que es inconsciente y expresa estados afectivos.

Las primeras palabras que comprende las emplea también para expresar estados afectivos y especialmente deseos. No son como se cree palabras para denominar objetos. Cuando dice "pan" no es que nombre indiferentemen- 
te el pan, sino que "quiere pan". Que el niño emplee una misma palabra para expresar cosas diversas, no significa que emplee conceptos generales, son simples expresiones de afectos o deseos al divisar cosas parecidas; por esto se equivoca tan a menudo.

Al año y medio puede nombrar lo que le rodea. Ya es la palabra el símbolo del objeto. Este primer lenguaje es concreto por excelencia. Al año y medio comienza a diferenciar las consonantes; del año y medio a dos años comienza la fase interrogativa, el uso de verbos en infinitivo; de sustantivos en nominativo y de adjetivos en positivo. Comienza a usar la conjunción “i”. Rara vez emplea antes de los dos años palabras negativas (jamás nunca). A los 2 años tiene el niño la comprensión de un vocabulario de 300 a 700 palabras.

Como hemos visto, hasta el año y medio se desarrolla especialmente las percepciones sen-

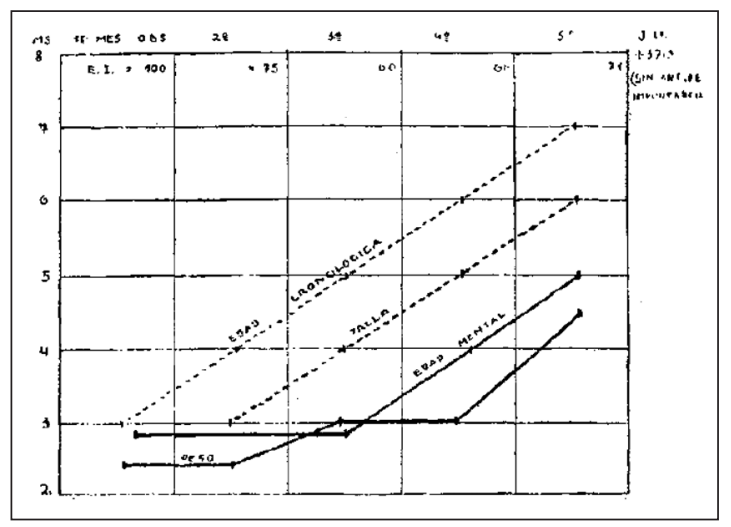

Figura 1.

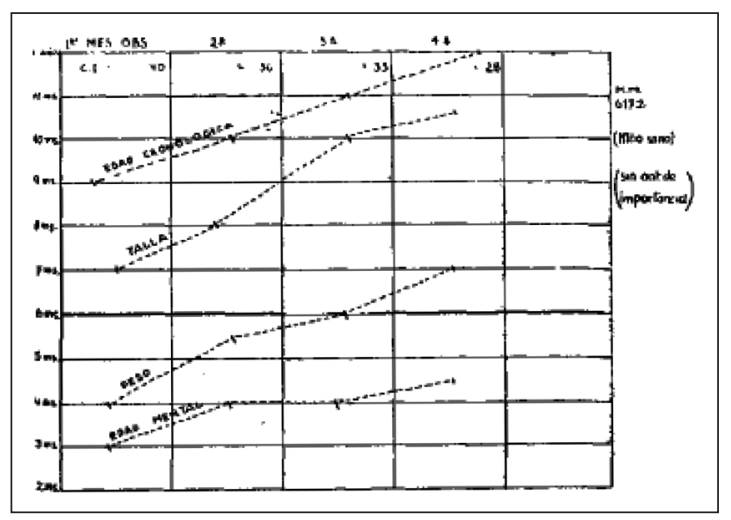

Figura 2. soriales y el control del movimiento; predominan los reflejos y los procesos instintivos. Pero ya al año se advierten los primeros síntomas de la inteligencia superior, como son los juicios y razonamientos. Se le ocurre, por ejemplo, servirse de herramientas para alcanzar algo. Esta etapa se ha llamado de chimpancé, por la analogía de conducta que observa con estos antropoides.

El desarrollo de la ideación aumenta rápidamente con la adquisición del lenguaje, aunque primero existe lo que se llama ideación muda, o sea, expresada mímicamente.

Los primeros juicios del niño son casos particulares y antes de los 4 años hay sólo conclusiones por analogía (abriga la muñeca, le da de comer a un juguete que representa un animal, buscándole el hocico, etc.)

Hasta los 3 años la inteligencia es práctica y egoísta, gira alrededor de la satisfacción de sus instintos.

En el lactante no hay imaginación puesto que no hay experiencia, y lo que a esta edad aparece como tal se debe a que para el niño pequeño todo es real. De ahí que la asociación, de casualidad domina el antropomorfismo (una silla le pegó, una almohada duerme, etc.)

Incluimos los gráficos obtenidos de un grupo de 30 niños que se crían en la sala que tengo a mi cargo en la Casa Nacional del Niño. A pesar de no poder dar aun cifras estadísticas exactas sobre el índice de inferioridad, debido a la escasez de material recolectado y al poco tiempo trascurrido desde que he empezado

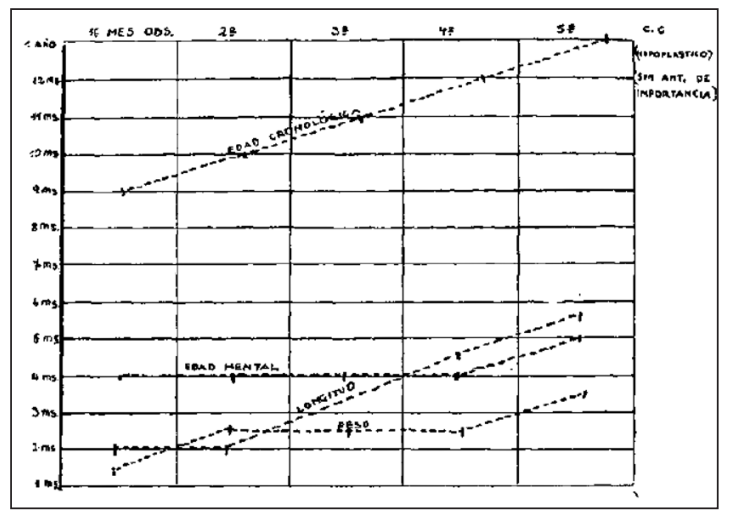

Figura 3. 
esta tarea, se puede apreciar el enorme atraso de desarrollo global que sufren estos niños. De paso diré que si bien es cierto que nuestro medio cuenta con un mayor porcentaje de tarados, tampoco es menos cierto que el retraso alcanza tanto a los tarados como a los indemnes de taras, cierto que en un grado un poco menor a estos últimos. Son estos, nuevos problemas que podrían esclarecerse en el futuro.

Diré también que en la Casa Nacional del Niño se han hecho muchas enmiendas y mejoras y que se podrán hacer muchas más aún; pero en el fondo, el problema subsiste. El orfelinato es un armatoste de otros tiempos de la era premicrobiana, de la era de la pecaminosidad del hijo natural y de otras mil consideraciones añejas, de orden ético social y técnico médicos.

\section{Comentario al artículo de Zorrilla}

Entre los siglos XVIII y XIX, diversos científicos comenzaron a estudiar la infancia a través de observaciones directas, habitualmente basadas en sus propios hijos. A partir de dichas observaciones, se construyeron escalas para la evaluación del desarrollo y la inteligencia de los niños, siendo las primeras escalas las de Alfred Binet en Francia y posteriormente, de Arnold Gesell en Estados Unidos ${ }^{6}$.

Desde las investigaciones más pioneras se vislumbraba la compleja interacción entre la naturaleza (biología y genética) y la nutrición (alimentos y afecto) en el desarrollo de los niños. "Herencia, enfermedades y un ambiente inadecuado" 7 sin duda se conjugaban amenazantes para los niños institucionalizados y también para aquellos niños que crecían en medio de precarias condiciones de un Chile empobrecido tras la Depresión de 1929, luego del derrumbe de la principal actividad económica, como era la exportación de salitre y cobre $^{8}$.

Probablemente la ola de cesantía, pobreza y desazón en que se sumó el país en los años 30, fueron determinantes del "brusco descenso" en las tasas de natalidad, "que el año 1931 alcanzó a 34,6 por 1,000 habitantes, cifras a que solo habiamos bajado durante el quinque- nio 1885-1889" (Calvo Mackenna 1932) ${ }^{9}$ y el marcado aumento de los niños nacidos fuera de matrimonio; hacia el año 1940 se estimaba que $37 \%$ de la población infantil era ilegíti$\mathrm{ma}^{2}$, tasa que si bien habría descendido al compararla con principios de siglo ${ }^{10}$, seguía siendo muy superior a países desarrollados, e incluso a otras naciones de la región ${ }^{2}$. Para ayudar a que los niños tuvieran a un buen nacer, a nivel nacional se desplegaron importantes campañas de educación sexual, de promoción de la puericultura y de legalización de los nacimientos y uniones físicas ${ }^{2,11,12}$.

No obstante los esfuerzos, las posibilidades de dar a los huachos una crianza digna eran limitadas; estos niños "poblaban los asilos, orfelinatos y cárceles" $" 13$, pesar de la elevada demanda por ingresar a las casas de huérfanos ${ }^{14}$, "Jamás era necesario rechazar una solicitud de ingreso" puesto que "De cuando en cuando epidemias de sarampión o coqueluche dejaban numerosas camas listas para recibir nuevos ingresados" (Ariztía y Eggers 1942) ${ }^{15}$.

Considerando las condiciones monótonas en que se criaban los niños institucionalizados, que distaban mucho de lo óptimo, era esperable la alta prevalencia de alteraciones del desarrollo" "El orfelinato es un armatoste de otros tiempos de la era premicrobiana, de la era de la pecaminosidad del hijo natural y de otras mil consideraciones añejas, de orden ético social y técnico médicos.”(Zorrilla 1934). “... el hospitalismo reinaba en esos servicios en todo su apogeo... lo cual acarrea a la larga perturbaciones en primer término en la psiquis del niño, que se traduce en anorexias rebeldes, retardo en sus funciones psíquicas y estáticas, distrofias, etc" (Ariztía 1935) ${ }^{16}$.

La "atrofia de la personalidad" secundaria a "ese ambiente anormal en donde, quiera o no, cada niño no es más que un número que completa un pequeño y grande rebaño humano", era una de las más temidas secuelas ${ }^{16,17}$. Por otro lado "Hemos visto volver después de algunos meses, rozagantes y hermosos a chicos que salidos del hospital en condiciones de lastimosa consunción se repusieron aun en condiciones desastrosas de higiene y de cuidados, únicamente cuando volvieron a hallar el ambiente cariñoso y acogedor del humilde re- 
gazo maternal." (Cienfuegos E. 1933) ${ }^{18}$. Esta descripción podría coincidir con el hospitalismo o Depresión Anaclíctica descrita por Spitz en los años 40, al estudiar la importancia de la interacción la madre y hijo para el desarrollo y bienestar infantil ${ }^{19}$.

Al respecto, el año 1941, Norma King referiría "El niño nace con cierto grado de capacidad mental y desarrollará un grado de inteligencia correspondiente a esta capacidad natural, siempre que las condiciones del medio ambiente le proporcionen los estímulos adecuados para su desarrollo. La sociedad debe tratar por todos los medios de colocar al niño en condiciones de que su inteligencia se desarrolle normalmente"

Consiente de las sub-óptimas condiciones en que se encontraban los niños, Zorrilla los observaba y día a día registraba sus progresos. Probablemente los hitos descritos en el presente artículo histórico no han variado, pero sin duda ha experimentado importantes cambios el entorno en que ocurre la crianza, la empatía e interpretación de las conductas del niño.

Gracias a las políticas de protección de la infancia que comenzaron a implementarse en el país en forma sistemática, organizada y sostenida hace prácticamente un siglo, se ha logrado un importante progreso a nivel nacional, no tan sólo en términos de mortalidad y desnutrición infantil, sino también en oportunidades, derechos, desarrollo y acceso a la educación.

\section{Referencias}

1.- Rojas J: Los derechos del niño en Chile: una aproximación histórica, 1910-1930. Historia (Santiago) 2007; 40 (1): 129-64.

2.- Illanes MA: Cuerpo y sangre de la política. La construcción histórica de las visitadoras sociales (1887-1940), Santiago, LOM Ediciones, 2007.

3.- Calvo Mackenna L: A Propósito de la Casa Nacional del Niño. Anuario Médico Social 1935; 7-12. Reimpreso en Rev Chil Pediatr 2010; 81 (4): 300-3.

4.- Baeza Goñi A: Algunas obras Privadas de Protección a la infancia en Chile. Rev Chil Pediatr 1933; 4.

5.- Zorrilla M: La Casa Nacional del Niño, algunos de sus problemas. Talleres Gráficos de la Casa Nacional, Santiago de Chile 1942.

6.- Santrock J: Psicología del Desarrollo en la infancia $7^{\circ}$ edición. McGraw Hill/Inteeramericana de España S.A.U., España 2003.

7.- King N: Test de Gessell en Lactantes. Rev Chil Pediatr 1941; 12: 21-36.

8.- Memoria Chilena: [consultado 12.12.13] Disponible en http://www.memoriachilena.cl/602/w3-article-601.html

9.- Calvo Mackenna A: Natalidad, mortinatalidad y mortalidad precoz en el recién nacido. Rev Chil Pediatr 1932; 3: 261-7.

10.- Salazar G: Ser Niño "Huacho" en la Historia de Chile (siglo XIX). Santiago. LOM Ediciones 2006.

11.- Schonhaut L, Millán T: La educación sexual debe comenzar en el hogar y continuar en la escuela. Comentario del artículo publicado en Revista Chilena de Pediatría 1931. Rev Chil Pediatr 2011; 82 (5): 454-60.

12.- Schonhaut L: Cartilla de Puericultura de la Sociedad Chilena de Pediatría. Rev Chil Pediatr 2008; 79 (1): 85-9.

13.- Álvarez G: El Servicio Social ante el problema de la madre soltera. Ministerio de Educación Pública. Escuela de Servicio Social, Memoria de Prueba para optar al título de Asistente Social del Estado de Santiago. 1944. En Illanes MA: Cuerpo y sangre de la política. La construcción histórica de las visitadoras sociales (1887-1940), Santiago, LOM Ediciones, 2007.

14.- Schonhaut L: Profilaxia del abandono cien años de protección de la infancia en Chile. Rev Chil Pediatr 2010; 81 (4): 301-9.

15.- Ariztia A, Eggers F: Alimentación artificial del lactante sano en establecimientos cerrados. Rev Chil Pediatr 1942; 13 (2-3): 73-93.

16.- Ariztía A: Anuario Médico de la Casa nacional del Niño. 1935.

17.- Cienfuegos E, Schonhaut L: Distrepsias de Origen Psíquico: Publicado en Rev Chil de Pediatr 1930: 1 (1): 6-10. Rev Chil Pediatr 2007; 78 (5): 542-8.

18.- Cienfuegos E: Algunos rasgos de la personalidad infantil. Rev Chil Pediatr 1933: 59-73.

19.- Spitz RA: El primer año de vida del niño. Fondo de la Cultura Económica. Primera Edición en Inglés 1965. Decimotercera reimpresión, Argentina, 2006. 